\title{
Marketing trifft Forschung
}

\section{ZIELGRUPPENORIENTIERTE DIGITALISIERUNG WISSENSCHAFTLICHER INHALTE}

Johannes Ebner [1], Peter Färberböck [1] \& Stefan Schwaiger [2]

[1] FB Geschichte, Universität Salzburg

johannes.ebner@marketext.at

peter.faerberboeck@marketext.at, https://orcid.org/0000-0002-8701-8259

[2] FB Germanistik, Universität Salzburg

stefan.schwaiger@marketext.at

www.marketext.at

Keywords: Content Marketing, Science Marketing, Audience-Oriented Digitalization

\begin{abstract}
Since the Bologna Process, the competition in higher education grew harsher. Thus, a target group oriented marketing approach in websites is desperately needed. This article depicts the approach of the Department of History of the University of Salzburg.
\end{abstract}

\section{Einleitung}

Im Zuge dieser Arbeit können wir nicht auf alle Gegebenheiten, wie Suchmaschinenoptimierung (SEO), Usability (Stichwort User Experience / UX) und Konkurrenzanalyse eingehen, da dies den Rahmen deutlich sprengen würde. Deswegen wird ein Überblick über die Projektarbeit der Website des Fachbereichs Geschichte gegeben und insbesondere auf am Anfang unvorhersehbare Herausforderungen und Besonderheiten eingegangen.

Konkret überarbeiten wir die Website des Fachbereichs Geschichte der Universität Salzburg. Dabei kam es zu einigen Herausforderungen, die von vornherein nicht offensichtlich waren. Im nächsten Kapitel werden einige dieser Herausforderungen vorgestellt und anschließend die ergriffenen Maßnahmen beschrieben. Am Beginn fokussieren wir uns jedoch auf die Gründe für zielgruppenorientierte Digitalisierung.

\section{Die Ausgangslage: Warum eigentlich Wissenschaftsmarketing?}

Institutionen haben gegenwärtig ein Problem, das gleichzeitig eine Chance darstellt: Sie können wissenschaftliche Inhalte und Erkenntnisse vermarkten oder nicht. Hier wiederum taucht ein „Warum“ auf. Warum sollte man wissenschaftliche Desiderata und Erkenntnisse mit Content-Marketing-Maßnahmen präsentieren? Eine kurze Erklärung: Content-Marketing vermittelt „Inhalte, die informierend, beratend und unterhaltend sind"1, und bietet so einen Mehrwert für Kund*innen, in diesem Fall angehende Studierende oder wissenschaftlichen Nachwuchs. Eine Antwort auf dieses „Warum“ liegt hier in der Ver-

1 Lutz FRÜHBRODT, Content Marketing. Wie „Unternehmensjournalisten“ die öffentliche Meinung beeinflussen, Frankfurt am Main 2016, 7, online unter: https://www.otto-brenner-stiftung.de/fileadmin/user_data/stiftung/02_Wissenschaftsportal/03_ Publikationen/AH86_Contentmarketing_Fruehbrodt_2016_06_09.pdf (04.04.2019). 
einheitlichung durch den Bologna-Prozess ${ }^{2}$, durch den neue Ausbildungsinstitutionen entstanden sind und sich gleichzeitig der wissenschaftliche Nachwuchs verringert.

Der Wettbewerb um die Bildung ist in vollem Gange. Dieser Wettbewerb erfordert eine Legitimation der Institutionen und Fachbereiche. Um Legitimation zu erlangen, braucht es eine Informationsoffensive.

Wettbewerb erfordert Legitimation.

Legitimation erfordert Information.

In der Studie create your UNIverse ${ }^{3}$ wurden Studierende befragt, was sie von Hochschulen und Forscher*innen erwarten. Aus insgesamt 2.105 Beiträgen (auch in sozialen Medien) von 754 Teilnehmer*innen folgerten sie fünf Handlungsempfehlungen für die künftige Gestaltung der Hochschule: ${ }^{4}$

- Digitalisierung besser nutzen

»Proaktiv und strategisch sowie virtuell und physisch

- Weiterentwicklung der Lehrenden

»Entwicklung von reinen Wissensvermittler*innen zu Lerncoaches und Mentor*innen

- Bildung von Strukturen, die Vernetzung und Lifelong-Learning ermöglichen

»Vernetzung von Studierenden, Alumni, Lehrenden, aber auch Unternehmen, NGOs und dem öffentlichen Sektor

- Alternative Lehrveranstaltungen

» Experimentierräume für alle Studierenden in der Lehre, die Interaktivität fördern. Gemeinsam forschen

- Ausbau internationaler und nationaler Zusammenarbeit

» Digital und nicht-digital

Diese Empfehlungen sind nur ein Ausblick in die Zukunft, was möglich wäre und wohin es, zumindest aus Sicht der Studienautor*innen, gehen soll. Dazu braucht es hingegen von der Seite der Universität, des Instituts oder des Fachbereichs einen stimmigen Markenauftritt, der Besucher*innen überzeugen soll. Ein Ausschnitt dieses Markenauftritts ist die Kommunikation nach außen und damit die inhaltliche Aufbereitung - das Content-Marketing - der Website.

Kurz: Marketing ist nötig, wo eine Wettbewerbssituation herrscht. Darunter fällt auch das angesprochene Content-Marketing.

Um zurück zum zentralen Punkt der Legitimation zu kommen, gilt es, diese Hinweise als Lösungsansätze im Rahmen der angesprochenen Legitimation zu sehen. Durch die aufbereiteten Inhalte, die mit diesem Ziel im Fokus entstanden sind und weiterhin entstehen (Inhalte bleiben nicht für immer

2 N.N., European Higher Education Area and Bologna Process, online unter: http://www.ehea.info/ (04.04.2019) und ausführlicher in Lena BECKER, Bildung im Zeichen der Ökonomisierung. Der Bologna-Prozess und seine Auswirkungen auf die Erziehungswissenschaft, Darmstadt 2012.

3 Im Auftrag der Ludwig Boltzmann Gesellschaft und des austrian council, durchgeführt durch das Consulting-Unternehmen winnovation consulting gmbh.

4 Vgl. Gertraud LeImüLleR u. a., create your UNIverse. Erwartungshaltungen Studierender an die Hochschulen der Zukunft, Wien 2017, 33-35, online unter: https://www.rat-fte.at/files/rat-fte-pdf/publikationen/2017/170427_Create\%20your\%20 UNIverse_finale\%20Praesentation.pdf (04.04.2019). 
stehen, sondern werden stets angepasst), sollen sich die ausgewählten Zielgruppen angesprochen fühlen. Sie werden dadurch ermuntert, sich individuell mit diesen auseinanderzusetzen.

\section{Mannigfaltige Herausforderungen - Von Stakeholdern bis zu Bildrechten}

Eines der Grundprobleme aller größeren Projekte - auch eine Neuaufstellung einer Fachbereichswebsite ist als großes Projekt zu sehen und war auch als solches geplant - ist die Absprache mit den Stakeholdern. Allgemein ist ein Stakeholder eine Gruppe oder eine Person, die ein berechtigtes Interesse am Verlauf oder Ergebnis eines Prozesses oder Projektes hat. ${ }^{5}$ Dabei ist nicht nur das umgangssprachliche ,mit ins Boot holen' gemeint, sondern auch die genaue Abstimmung und transparente Kommunikation mit allen Beteiligten. Hier fällt auch die zeitliche Terminierung ins Gewicht, denn je mehr Stakeholder ein Projekt hat, desto komplexer wird es, Lösungsansätze in den Tagesablauf einzubinden. Hier kann es zu Zeitverzögerungen kommen - vor allem bei der Terminierung von größeren Meetings oder der Absprache mit mehreren Stakeholdern.

Im Fall der Website des Fachbereichs Geschichte wurde dies über eine Arbeitsgruppe Website (aus ursprünglich acht Personen) abgewickelt, die sich regelmäßig traf. Hier informierten wir die Gruppe über den Projektstand und konnten unmittelbar Feedback einholen und entsprechend nachkorrigieren. Der Fachbereichsrat, der aus dem Vorsitzenden, Vertreter*innen der Professor*innen, des akademischen Mittelbaus, der Studierenden, des allgemeinen Universitätspersonals, der Personalvertretung und dem Arbeitskreis für Gleichbehandlungsfragen besteht, diente als Lenkungsausschuss. ${ }^{6}$

Eines der prägenden Stichwörter der Organisation war die verteilte Verantwortlichkeit. Texte der Website, die eigentlich auf derselben Informationsebene existierten, z. B. Beschreibungen von zwei Forschungssäulen ${ }^{7}$ oder Kernfächern, hatten verschiedene Verantwortliche. Dies ist kein Novum, sondern bei der ,natürlichen' Entstehungsgeschichte einer Seite sehr oft die Norm. Durch die verteilte Verantwortlichkeit waren die Texte jedoch uneinheitlich, weil die Absprache untereinander fehlte. Durch die fließende Entstehung der Inhalte gab es Unterschiede in der Informationsdichte, aber auch größere Abweichungen in Länge, Aufbau und auch Gliederung. Diese größere Inkohärenz ist bei einem Webauftritt, der möglichst konsistent und stringent wirken sollte, ein größeres Problem und vermittelt unfreiwillig Unprofessionalität.

Zusätzlich zu den internen Abstimmungsaufgaben des Projekts war der allgemeine Aufbau der Website ein Arbeitspaket. Die Fachbereichsseite unterliegt der Corporate Identity der Website der Universität Salzburg und somit auch dem allgemeinen Aufbau dieser. Dies äußert sich dadurch, dass der Aufbau und auch der Inhalt einer Seite nicht komplett vom Fachbereich bestimmt werden kann. Im Gegenteil: Nur der mittlere Bereich kann befüllt werden, die außenliegenden Bilder, News-Spalten oder das Menü sind starr vorgegeben. ${ }^{8}$

5 Sonja EILmAnn u. a., Interessengruppen / Interessierte Parteien, in: Michael Gessler, Hg., Kompetenzbasiertes Projektmanagement. Band 1, Nürnberg 2011, 71.

6 Vgl. N. N., Fachbereichsrat des Fachbereichs Geschichte, online unter: https://uni-salzburg.at/index.php?id=23305 (04.04.2019).

7 Forschungssäulen sind Forschungsgruppen des Fachbereichs, auf die sich die einzelnen Forschenden in Gruppen spezialisiert haben, vgl. N. N., Forschungssäulen. Fachbereich Geschichte, online unter: https://uni-salzburg.at/index. php?id=202971 (04.04.2019).

8 Vgl. N. N., Kernfächer und Arbeitsbereiche. Fachbereich Geschichte, online unter: https://uni-salzburg.at/index. php?id=23385 (04.04.2019). Hier kann nur der mittlere Text geändert werden und höchstens mit Bildern angereichert 


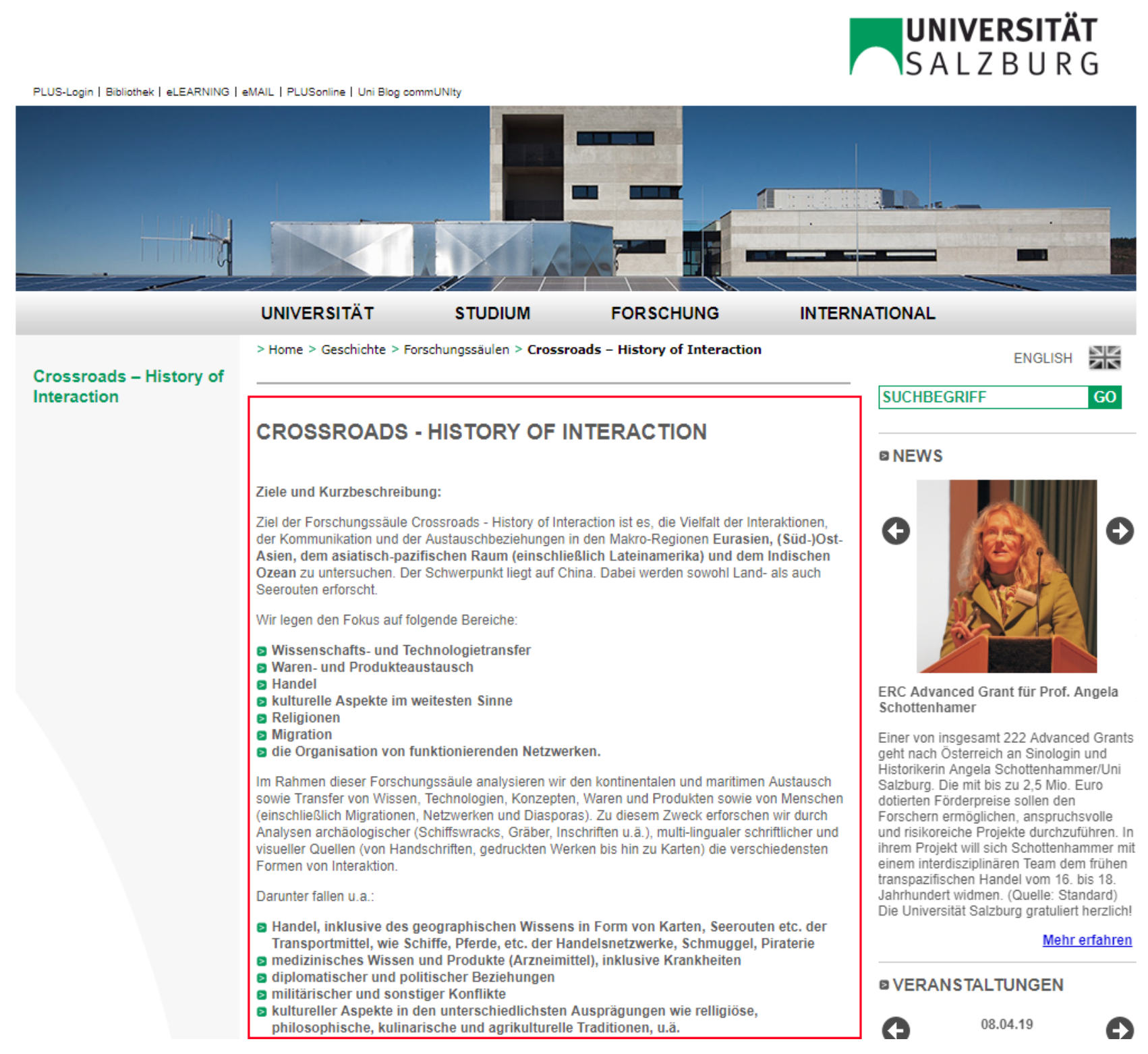

Abb. 1: Eigener Screenshot, Website des Fachbereichs Geschichte. Der mittlere Teil, der befüllt werden kann, ist rot markiert.

Die Verantwortung der einzelnen Websites oblag dem wissenschaftlichen Personal, was zu einem weiteren Problem führte: Die Sprache im Content-Marketing ist einfacher, schnell verständlich und auch mit weniger Aufmerksamkeit lesbar. Dazu soll sie begeistern. Die vorher genutzte wissenschaftliche Sprache war hingegen deutlich komplexer. Unsere Aufgabe war es, diese zwei Welten zu vereinen: Die ausgewiesene Fachkunde weiterhin im Vordergrund zu behalten und gleichzeitig attraktiv die Zielgruppen zu bedienen. Hier war eine Herausforderung, die Verantwortlichen davon zu überzeugen, dass kurze, einfache Texte mit stärkerer Bebilderung das probate Mittel für die Fachbereichswebsite sind. Der PR-Effekt der Seite musste erst ins Bewusstsein gerufen werden. Die Inhalte wären nämlich öffentlichkeitswirksam, einzig die Präsentation musste angepasst werden. In diesem Zuge ist auch die Definition der Zielgruppe unumgänglich - die dazu definierten Personas sind im nächsten Kapitel beschrieben.

und verschönert werden. Der Bereich rundherum ist vorgegeben. 
Am Rande sind auch die Bildrechte eine Herausforderung. Verwendete Bilder und Videos werden deswegen zum Großteil vom Fachbereich selbst gemacht. Von den Beteiligten werden die Bildrechte schriftlich eingeholt. Bei den Stock-Fotos ${ }^{9}$ müssen diese gegebenenfalls gekauft werden, sofern sie nicht frei verwendbar sind.

\section{Planung, Zielfindung und Umsetzung der zielgruppenorientierten Digitalisierung}

In den folgenden Absätzen wird auszugsweise beschrieben, welche Grundmaßnahmen angewandt wurden, um so zur gewünschten zielgruppenorientierten Digitalisierung zu kommen. Wie angesprochen, war der Grundstein für die Planung die Gründung einer Task Force, die mit Schlüsselpersonen (Fachbereichsleitung, IT, Sekretariat) besetzt wurde. In dieser wurde die Planung und die Umsetzung des Projekts besprochen. Zusätzlich kam diese zu Workshops zusammen, wo grundlegende Richtungsentscheidungen festgelegt und z. B. Stakeholder, Personas und Konkurrenz zumindest grob identifiziert wurden.

Einer der nächsten Schritte war ein Content-Audit der Seite. Dieser stellt eine Art Prüfung aller derzeit verfügbaren Web-Inhalte einer Seite dar. ${ }^{10}$ Dabei wird die Website qualitativ ausgewertet und nach Verbesserungsbedarf gesucht. Jede einzelne Seite wurde kategorisiert (About, News, etc.), die potenzielle Zielgruppe der Seite identifiziert und die verwendeten Inhaltstypen aufgelistet. Aufgrund dessen wurde der Inhalt nach Qualität bewertet sowie positive und negative Merkmale hervorgehoben, sodass diese in die zukünftige Seite übernommen bzw. verhindert werden können.

Ein weiterer Kernschritt war die Erstellung von Personas. Diese sind fiktionale Figuren, die jeweils einen spezifischen Typ eines potenziellen Benutzers charakterisieren. ${ }^{11}$ Dazu werden grobe soziodemografische Daten der jeweiligen Zielgruppensegmente nötig, aber auch grobe Umschreibungen der Gefühlswelt, wie Ängste, die Lebenswelt und die Motivation, die die Person antreibt, eingearbeitet. Selbst ein simulierter ,Kaufprozess', d. h. wie würde er oder sie sich auf der Website fortbewegen und was sind seine Zielseiten, sowie die vermutete Mediennutzung (z. B. soziale Medien, Offline-Medien oder Handy-Nutzung) werden implementiert. Durch diesen genauen Steckbrief können Inhalte speziell für die Zielgruppensegmente erstellt werden.

Die deutlichen Einschränkungen durch die fixe Vorgabe der Universitäts-Website führte zu Kompromissen und auch anderen Ideen. Eine davon ist eine eigene Seite für Studierende in Blog-Form basierend auf WordPress, die optisch und inhaltlich schneller und einfacher angepasst werden kann. Diese Flexibilität ermöglicht es erst, optisch und technisch dem schnelllebigen Zeitgeist des Internets nachzukommen. Diese Idee wird in naher Zukunft umgesetzt und befindet sich in der Endphase der Planung und Vorbereitung. Andere Bereiche bekommen zielgruppengerechte Texte und werden stärker bebildert. Auch an Video-Inhalten wird derzeit gearbeitet - diese befinden sich noch in der Konzeptphase.

$9 \quad$ Stock Fotos sind in Datenbanken erhältlich und je nach Benutzung unterliegen sie verschiedenen Lizenzen. Sie können kostenlos oder gebührenpflichtig sein. Vgl. Michael R. PERES, The Focal Encyclopedia of Photography. Digital Imaging, Theory and Applications, History and Science, New York / London 2007, 351.

10 Vgl. Kristina HALVORSON / Melissa RACH, Content Strategy for the Web, Berkeley 2012, 46-67. Unsere Vorgehensweise wird grob in folgendem Blogpost skizziert: Johannes EBNER / Peter Färberböck / Stefan SchWAIGER, Die Definition des Content Audits, online unter: https://www.marketext.at/blog/definition-des-content-audits/ (04.04.2019).

11 Vgl. Jill ButLeR / Kritina HoLden / William LIDWELL, Universal Principles of Design. A Cross-disciplinary Reference, Beverly $2010,182$. 


\section{Fazit}

„Eine Website ist doch schnell gemacht. Die Texte schreiben wir selbst - als Historiker*innen ist das unser tägliches Brot«. Genau so könnte man sich die Ausgangslage vorstellen, wenn eine Fachbereichsseite erstellt wird. Diese Annahme ist nicht völlig falsch, denn tatsächlich ist die Verschriftlichung ein großer Teil des Historikerberufes. Dabei werden aber einige Dinge übersehen, denn auch wissenschaftliche Papers haben eine definierte Zielgruppe, für die man schreibt. So funktioniert es auch bei Web-Texten. Dieses Projekt stellt dabei den Grundplan für einen sich stetig ändernden Prozess dar. Die Task Force, die Personas und die Veränderung der technischen Möglichkeiten beeinflussen diesen stets. Es steht am Ende kein fertiger digitaler Auftritt, der über Jahre gültig ist, sondern vielmehr ein Gerüst, das stets gepflegt und angepasst werden muss. Ein solcher Prozess ist inhärent nötig, um in der heutigen Konkurrenzsituation als wissenschaftliche Institution zu überleben.

\section{Literaturverzeichnis}

Lena BECKER, Bildung im Zeichen der Ökonomisierung. Der Bologna-Prozess und seine Auswirkungen auf die Erziehungswissenschaft, Darmstadt 2012.

Jill Butler / Kritina Holden / William LidwelL, Universal Principles of Design. A Cross-disciplinary Reference, Beverly 2010.

Johannes Ebner / Peter Färberböck / Stefan Schwalger, Die Definition des Content Audits, online unter: https:// www.marketext.at/blog/definition-des-content-audits/ (04.04.2019).

Sonja Elımann u. a., Interessengruppen / Interessierte Parteien, in: Michael Gessler, Hg., Kompetenzbasiertes Projektmanagement. Band 1, Nürnberg 2011.

Lutz FrüHBRODT, Content Marketing. Wie „Unternehmensjournalisten“ die öffentliche Meinung beeinflussen, Frankfurt am Main 2016, online unter: https://www.otto-brenner-stiftung.de/fileadmin/user_data/stiftung/02_ Wissenschaftsportal/03_Publikationen/AH86_Contentmarketing_Fruehbrodt_2016_06_09.pdf (04.04.2019).

Kristina HALVORSON / Melissa RACH, Content Strategy for the Web, Berkeley 2012.

Gertraud LEIMÜLLER U. a., create your UNIverse. Erwartungshaltungen Studierender an die Hochschulen der Zukunft, Wien 2017, online unter: https://www.rat-fte.at/files/rat-fte-pdf/publikationen/2017/170427_Create\%20 your\%20UNIverse_finale\%20Praesentation.pdf (04.04.2019).

N. N., European Higher Education Area and Bologna Process, online unter: http://www.ehea.info/ (04.04.2019).

N. N., Fachbereichsrat des Fachbereichs Geschichte, online unter: https://uni-salzburg.at/index.php?id=23305 (04.04.2019).

N. N., Forschungssäulen. Fachbereich Geschichte, online unter: https://uni-salzburg.at/index.php?id=202971 (04.04.2019).

N. N., Kernfächer und Arbeitsbereiche. Fachbereich Geschichte, online unter: https://uni-salzburg.at/index.php?id=23385 (04.04.2019).

Michael R. Peres, The Focal Encyclopedia of Photography. Digital Imaging, Theory and Applications, History and Science, New York / London 2007. 